\title{
Análise de Parâmetros na Modelagem de Malária
}

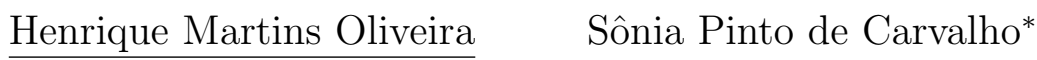 \\ Departamento de Matemática - ICEx - UFMG
}

Em [1], Rodriguez, Delgado, Ramos, Weinberg e Rangel propõem o modelo abaixo para a dinâmica da epidemia de malária na Península de Paria na Venezuela:

$$
\begin{aligned}
\frac{d X(t)}{d t} & =\beta(N-X(t)) Y(t)-\gamma X(t) \\
\frac{d Y(t)}{d t} & =\beta(M(t)-Y(t)) X(t)-m Y(t) \\
\frac{d M(t)}{d t} & =\left(\frac{\nu_{1}}{1+\nu_{2} L(t)}\right) L(t)-m M(t) \\
\frac{d L(t)}{d t} & =b M(t)-\left(\frac{\nu_{1}}{1+\nu_{2} L(t)}+\mu\right) L(t)
\end{aligned}
$$

onde $M$ é a população de mosquitos, $L$ é a população de larvas, $X$ a população de humanos infectados, $Y$ a de mosquitos infectados e as outras letras são parâmetros do problema.

No mesmo artigo, mostram que existe um equilíbrio para a população dos mosquitos sem a presença da infecção, tanto entre mosquitos como entre humanos, e que é linearmente estável para os valores dos parâmetros calculados especificamente para a Península de Paria.

No nosso trabalho, mostramos que existe uma região no espaço de parâmetros que contém os valores calculados pelos autores e onde o equilíbrio sem infecção estudado é linearmente estável.

Também estudamos a bacia de atração desse equilíbrio linearmente estável. Nas variáveis $(M, L)$ o sistema desacopla e a bacia contém todos os valores possíveis de $0<L$ e $0<M$. No caso do espaço de dimensão 4 , observamos através de simulações numéricas que a bacia é todo o espaço de fase.

Dessa análise, surgem algumas conclusões contra-intuitivas como, por exemplo, os possíveis malefícios causados por uma diminuição aguda no tempo de recuperação dos doentes na estabilidade do equilíbrio que nos interessa.

Palavras-chave: malária, modelagem matemática, estabilidade de equilíbrios

*Orientadora 


\section{Referências}

[1] Diego J. Rodríguez; Laura Delgado; Santiago Ramos; Vanessa Weinberger; Yadira Rangel. A model for the dynamics of malaria in Paria Peninsula, Sucre State, Venezuela. Ecological Modelling, 259:1-9, 2013.

[2] Morris W. Hirsch; Stephen Smale; Robert L. Devaney. Differential Equations, Dynamical Systems, and an Introduction to Chaos. Academic Press, 2004. 